\title{
Synthesis, Characterization and Molecular Docking of Novel Quinoline and Pyridine Derivatives
}

\section{SUHEB SALEH ${ }^{1}$, USAMA AL-TIMARI ${ }^{2}$, AEED AL- FAHDAWI ${ }^{3}$, NASSEEM EL-KHATATNEH ${ }^{4}$, CHANDRA $^{5}$, MAHENDRA $\mathrm{M}^{4}$ and MOHAMMED AL-GHORBANI ${ }^{6, *}$}

'Department of Chemistry, Al Rasafi School, Ministry of Education , Anbar, Iraq.

2Department of Chemistry, College of Health Medical Technology, Baghdad, Iraq.

${ }^{3}$ Department of Chemistry, College of Education for Women , University of Anbar, Iraq. ${ }^{4}$ Department of Studies in Physics, Manasagangotri, University of Mysore, Mysore-570006, Karnataka, India.

${ }^{5}$ Department of Physics, The National Institute of Engineering (NIE), Mysore-570008, Karnataka, India.

${ }^{6}$ Department of Chemistry, College of Education, University of Thamar, Yemen.

*Corresponding author E-mail: malghorbani@yahoo.com

http://dx.doi.org/10.13005/ojc/330604

(Received: August 01, 2017; Accepted: August 25, 2017)

\begin{abstract}
A new series of substituted quinoline and pyridine derivatives $6 a-h$ are synthesized by the coupling of hydrazide derivatives $4 a-b$ with substituted carboxylic acids $5 a-c$ in the presence of $\mathrm{N}, \mathrm{N}, \mathrm{N}$ ',N'-tetramethyl-o-(benzotriazol-1-yl) uranium tetrafluoroborate TBTU as a coupling agent and lutidine as a base. The newly synthesized compounds $6 \mathrm{a}-\mathrm{I}$ is characterized by analytical NMR and mass spectral data. The newly synthesized compounds were subjected to molecular docking studies for the inhibition of human Aurora A kinase target to evaluate their potential value for the treatment of cancers.
\end{abstract}

Keyword: Synthesis, Molecular docking, Quinoline, Pyridine, NMR and Mass spectral.

\section{INTRODUCTION}

Quinoline nucleus represent a significant class of heterocycles, they occur in various natural products, especially in alkaloids and exhibit exceptionally broad spectrum of biological activities ${ }^{1-3}$. The synthesis of quinoline and their derivatives has attracted considerable attention from organic and medicinal chemists for many years as a large number of natural and synthetic products contain this heterocyclic nucleus ${ }^{4-8}$. Some derivatives containing quinoline ring system have been shown to possess useful biological activities, such as antiprion ${ }^{4}$, antimicrobial| ${ }^{5-8}$, antibacterial ${ }^{9}$, antitubercular ${ }^{10-12}$ and anticancer activities ${ }^{13-15}$. Similarly, it is well documented that pyridine nucleus is associated with a variety of pharmacological actions, including, pathogenesis of cancer, HIV, autoimmune and inflammatory diseases. ${ }^{16,17}$

In view of the interest in the activity spectrum and profile of quinoline and pyridine, and in continuation of our work on the synthesis of new compounds of pharmacological and biological 
interes $^{18-22}$, we describe herein the preparation and Doking study of some new quinoline and pyridine derivatives (6a-h).

\section{MATERIAL AN METHODS}

\section{General methods}

Chemicals were purchased from Sigma Aldrich Chemical Co. TLC was performed on aluminium-backed silica plates and visualized by UV-light. Melting points were determined on a Thomas Hoover capillary melting point apparatus with a digital thermometer. IR spectra were determined on JASCO FT-IR 4000 spectrophotometers. NMR spectra were recorded on Agilent-400 NMR MHz spectrometer in $\mathrm{CDCl}_{3}$. Chemical shifts were recorded in parts per million downfield from tetramethylsilane. Mass spectra were carried out on LC-MS/MS (API-4000) mass spectrometer. The elemental analysis of the compounds was performed on a Perkin Elmer 2400 Elemental Analyzer. The results of elemental analyses were within $\pm 0.4 \%$ of the theoretical values.

\section{Chemical synthesis}

General synthesis of ethyl acetate analogues (3a-b)

A mixture of commercially available 8-hydroxy quinoline or 5-methyl-pyridin-2-ol 1a-b $(0.03 \mathrm{~mol})$ and chloroethyl acetate $2(0.045 \mathrm{~mol})$ in dry acetone $(30 \mathrm{~mL})$ with anhydrous potassium carbonate $(0.045 \mathrm{~mol})$ were refluxed for $10-16$ hours. The reaction mixture was cooled and solvent removed by distillation. The residual mass was triturated with cold water to remove potassium carbonate, and extracted with ether $(3 \times 30 \mathrm{~mL})$. The ether layer was washed with $10 \%$ sodium hydroxide solution $(3 \times 30 \mathrm{~mL})$ followed by water $(3 \times 30 \mathrm{~mL})$ and then dried over anhydrous sodium sulphate and evaporated to afford compounds 3a-c. The physical and analytical data of the synthesized title compounds $3 a-c$ are given as follows.

\section{4-(Quinolin-8-yloxy)-acetic acid ethyl ester (3a)}

Yield $90 \%$, yellow liquid, IR $\left(\mathrm{cm}^{-1}\right): 1735$ $(\mathrm{C}=\mathrm{O}), 3115-3225\left(\mathrm{NHNH}_{2}\right) ;{ }^{1} \mathrm{H}$ NMR $\left(\mathrm{CDCl}_{3}\right): \delta$ $(\mathrm{ppm})=2.36\left(\mathrm{t}, 3 \mathrm{H}, \mathrm{CH}_{3}\right), 3.28\left(\mathrm{q}, 2 \mathrm{H}, \mathrm{CH}_{2}\right), 4.21$ (t, 2H, OCH $)_{2}, 7.18-8.85$ (m, 6H, Ar-H). LC-MS m/z $=232$ Anal. Cal. for $\mathrm{C}_{13} \mathrm{H}_{13} \mathrm{NO}_{3}: \mathrm{C}, 67.52 ; \mathrm{H}, 5.67$. Found: C, $67.35 ; \mathrm{H}, 5.51 \%$.
(5-Methyl-pyridin-2-yloxy)-acetic acid ethyl ester (3b) Yield $87 \%$, yellow liquid, IR $\left(\mathrm{cm}^{-1}\right): 1745$ $(\mathrm{C}=\mathrm{O}) ; 3110-3215\left(\mathrm{NHNH}_{2}\right)^{1} \mathrm{H} \mathrm{NMR}\left(\mathrm{CDCl}_{3}\right): \delta$ $(\mathrm{ppm})=2.27\left(\mathrm{t}, 3 \mathrm{H}, \mathrm{CH}_{3}\right), 2.45\left(\mathrm{t}, 3 \mathrm{H}, \mathrm{CH}_{3}\right), 3.22$ (q, 2H, $\left.\mathrm{CH}_{2}\right), 4.29\left(\mathrm{t}, 2 \mathrm{H}, \mathrm{OCH}_{2}\right), 7.27-8.74(\mathrm{~m}, 3 \mathrm{H}$, Ar-H). LC-MS m/z =196 Anal. Cal. for $\mathrm{C}_{10} \mathrm{H}_{13} \mathrm{NO}_{3}: \mathrm{C}$, 61.53; $\mathrm{H}, 6.71$. Found: $\mathrm{C}, 61.38 ; \mathrm{H}, 6.54 \%$.

\section{General synthesis of hydrazide derivatives (4a-b)}

Hydrazine hydrate $(0.018 \mathrm{~mol})$ was added to a solution of compounds $3 a-b(0.018 \mathrm{moL})$ in absolute ethanol $(30 \mathrm{~mL})$ and continuously stirred for 2-4 h. at room temperature. A solid was separated out, which was quenched with water $(50 \mathrm{~mL})$, filtered and washed with water $(50 \mathrm{~mL})$. Finally, the solid was dried under vacuum and recrystallized with ethanol to obtain compounds $4 a-b$.

(Quinolin-8-yloxy)-acetic acid hydrazide (4a)

Yield $80 \%$, white solid $\mathrm{mp} ; 145-147^{\circ} \mathrm{C}$, IR $\left(\mathrm{cm}^{-1}\right): 1720(\mathrm{C}=\mathrm{O}) ;{ }^{1} \mathrm{H} \mathrm{NMR}\left(\mathrm{CDCl}_{3}\right): \delta(\mathrm{ppm})=4.45$ $\left(\mathrm{s}, 2 \mathrm{H}, \mathrm{NH}_{2}\right), 4.71\left(\mathrm{~s}, 2 \mathrm{H}, \mathrm{CH}_{2}\right), 7.21-8.87(\mathrm{~m}, 6 \mathrm{H}$, Ar), $9.41(\mathrm{~s}, 1 \mathrm{H}, \mathrm{NH}), \mathrm{LC}-\mathrm{MS} \mathrm{m} / \mathrm{z}=182$ Anal. Cal. for $\mathrm{C}_{8} \mathrm{H}_{11} \mathrm{~N}_{3} \mathrm{O}_{2}: \mathrm{C}, 53.03 ; \mathrm{H}, 6.12$. Found: C, 52.86; $\mathrm{H}, 6.03 \%$.

(5-Methyl-pyridin-2-yloxy)-acetic acidhydrazide (4b) Yield $87 \%$, white solid $\mathrm{mp} ; 178-180^{\circ} \mathrm{C}$, IR $\left(\mathrm{cm}^{-1}\right): 1735(\mathrm{C}=\mathrm{O}) ;{ }^{1} \mathrm{H} \mathrm{NMR}\left(\mathrm{CDCl}_{3}\right): \delta(\mathrm{ppm})=4.23$ $\left(\mathrm{s}, 2 \mathrm{H}, \mathrm{NH}_{2}\right), 4.83\left(\mathrm{~s}, 2 \mathrm{H}, \mathrm{CH}_{2}\right), 7.55-8.37(\mathrm{~m}, 3 \mathrm{H}$, Ar), 9.21 (s, $1 \mathrm{H}, \mathrm{NH}$ ); LC-MS m/z =218 Anal. Cal. for $\mathrm{C}_{11} \mathrm{H}_{11} \mathrm{~N}_{3} \mathrm{O}_{2}: \mathrm{C}, 60.82 ; \mathrm{H}, 5.10$. Found: C, 60.60; $\mathrm{H}$, $5.02 \%$.

\section{General synthesis of compounds (6a-h)}

Hydrazide derivatives $(\mathbf{4 a - b}, 3 \mathrm{mmol})$ in dry DCM $(20 \mathrm{ml})$ was stirred at $25-30^{\circ} \mathrm{C}$, and then lutidine ( $4 \mathrm{mmol}$ ) was added, followed by the addition of substituted amino-4-phenyl-1,3-thiazoles ( $3 \mathrm{mmol})$. The reaction mixture was stirred at the same temperature for $30 \mathrm{~min}$. then cooled to $0-5^{\circ} \mathrm{C}$ and TBTU $(3 \mathrm{mmol})$ was added over a period of 30 min maintaining the temperature below $5^{\circ} \mathrm{C}$. The reaction mass was stirred overnight and monitored by TLC using chloroform: methanol $(9: 1)$ as the mobile phase. The solvent was evaporated at reduced pressure, quenched by the addition of crushed ice and the obtained solid was filtered, dried and recrystallized from ethanol to afford compounds 6a-h in good yield. 
(4-Fluoro-phenyl)-acetic acid N'-[2-(quinolin-8yloxy)-acetyl]-hydrazide (6a)

Yield $76 \%$, white solid $\mathrm{mp} ; 255-257^{\circ} \mathrm{C}$, IR $\left(\mathrm{cm}^{-1}\right)$ 1640, $1675(\mathrm{C}=\mathrm{O}), 3245-3470(\mathrm{NH}-\mathrm{NH})$; ${ }^{1} \mathrm{H} \mathrm{NMR}\left(\mathrm{CDCl}_{3}\right): \delta(\mathrm{ppm})=3.21\left(\mathrm{~s}, 2 \mathrm{H}, \mathrm{CH}_{2}\right), 4.76$ (s, $\left.2 \mathrm{H}, \mathrm{CH}_{2}\right), 7.26-8.94(\mathrm{~m}, 10 \mathrm{H}, \mathrm{Ar}), 10.23(\mathrm{~d}, 2 \mathrm{H}$, 2NH); LC-MS m/z =354 Anal. Cal. for $\mathrm{C}_{19} \mathrm{H}_{16} \mathrm{FN}_{3} \mathrm{O}_{3}$ : C, $64.58 ; \mathrm{H}, 4.56$. Found: C, $64.37 ; \mathrm{H}, 4.80 \%$.

(4-Chloro-phenyl)-acetic acid N'-[2-(quinolin-8yloxy)-acetyl]-hydrazide (6b)

Yield $80 \%$, white solid, $\mathrm{mp} ; 258-260^{\circ} \mathrm{C}$, IR ( $\left.\mathrm{cm}^{-1}\right)$ : 1630, $1685(\mathrm{C}=\mathrm{O})$, 3255-3475 (NH-NH); ${ }^{1} \mathrm{H} \mathrm{NMR}\left(\mathrm{CDCl}_{3}\right): \delta(\mathrm{ppm})=3.44\left(\mathrm{~s}, 2 \mathrm{H}, \mathrm{CH}_{2}\right), 4.83$ (s, 2H, $\mathrm{CH}_{2}$ ), 7.21-8.85 (m, 10H, Ar), 10.24 (d, 2H, $2 \mathrm{NH}$ ), LC-MS m/z =370 Anal. Cal. for $\mathrm{C}_{19} \mathrm{H}_{16} \mathrm{CIN}_{3} \mathrm{O}_{3}$ : C, $61.71 ; \mathrm{H}, 4.36$. Found: C, 61.66; H, 4.17\%.

(3,4-Dichloro-phenyl)-acetic acid N'-[2-(quinolin8-yloxy)-acetyl]-hydrazide (6c)

Yield $73 \%$, white solid $\mathrm{mp} ; 228-230^{\circ} \mathrm{C}$, IR $\left(\mathrm{cm}^{-1}\right)$ : 1660, $1690(\mathrm{C}=\mathrm{O}), 3235-3455(\mathrm{NH}-\mathrm{NH})$; ${ }^{1} \mathrm{H}$ NMR $\left(\mathrm{CDCl}_{3}\right): \delta(\mathrm{ppm})=3.35\left(\mathrm{~s}, 2 \mathrm{H}, \mathrm{CH}_{2}\right), 4.69$ (s, 2H, $\mathrm{CH}_{2}$ ), 7.43-8.97 (m, 9H, Ar), 10.21 (d, 2H, $2 \mathrm{NH}$ ). LC-MS $\mathrm{m} / \mathrm{z}=405$ Anal. Cal. for $\mathrm{C}_{19} \mathrm{H}_{15} \mathrm{Cl}_{2} \mathrm{~N}_{3} \mathrm{O}_{3}: \mathrm{C}, 56.45 ; \mathrm{H}, 3.74$. Found: $\mathrm{C}, 56.32$; $\mathrm{H}, 3.51 \%$.

\section{4-Methoxy-benzoic acid N'-[2-(quinolin-8-yloxy)- acetyl]-hydrazide (6d) \\ Yield $78 \%$, white solid $\mathrm{mp} ; 253-255^{\circ} \mathrm{C}$, IR $\left(\mathrm{cm}^{-1}\right): 1635,1680(\mathrm{C}=\mathrm{O}), 3230-3470(\mathrm{NH}-\mathrm{NH}) ;{ }^{1} \mathrm{H}$ $\operatorname{NMR}\left(\mathrm{CDCl}_{3}\right): \delta(\mathrm{ppm})=3.30\left(\mathrm{~s}, 3 \mathrm{H}, \mathrm{CH}_{3}\right), 4.88$ (s, 2H, $\mathrm{CH}_{2}$ ), 7.37-8.74 (m, 10H, Ar), 10.27-10.34 (d, $2 \mathrm{H}, 2 \mathrm{NH}$ ). LC-MS $\mathrm{m} / \mathrm{z}=352$ Anal. Cal. for $\mathrm{C}_{19} \mathrm{H}_{17} \mathrm{~N}_{3} \mathrm{O}_{4}: \mathrm{C}, 64.95 ; \mathrm{H}, 4.88$. Found: $\mathrm{C}, 64.81$; $\mathrm{H}, 4.67 \%$.}

(4-Fluoro-phenyl)-acetic acid N'-[2-(5-methylpyridin-2-yloxy)-acetyl]-hydrazide (6e)

Yield $71 \%$, white solid $\mathrm{mp} ; 211-213^{\circ} \mathrm{C}$, IR $\left(\mathrm{cm}^{-1}\right): 1640,1695(\mathrm{C}=\mathrm{O}), 3250-3475(\mathrm{NH}-\mathrm{NH}) ;{ }^{1} \mathrm{H}$ $\operatorname{NMR}\left(\mathrm{CDCl}_{3}\right): \delta(\mathrm{ppm})=1.89\left(\mathrm{~s}, 3 \mathrm{H}, \mathrm{CH}_{3}\right), 3.34$ $\left.\mathrm{s}, 2 \mathrm{H}, \mathrm{CH}_{2}\right), 4.41\left(\mathrm{~s}, 2 \mathrm{H}, \mathrm{CH}_{2}\right), 6.18-7.69(\mathrm{~m}, 7 \mathrm{H}$, $\mathrm{Ar}), 10.13^{2}(\mathrm{~d}, 2 \mathrm{H}, 2 \mathrm{NH})$. LC-MS $\mathrm{m} / \mathrm{z}=318$ Anal. Cal. for $\mathrm{C}_{16} \mathrm{H}_{16} \mathrm{FN}_{3} \mathrm{O}_{3}: \mathrm{C}, 60.56 ; \mathrm{H}, 5.08$. Found: $\mathrm{C}$, $60.45 ; \mathrm{H}, 5.04 \%$.

(4-Chloro-phenyl)-acetic acid N'-[2-(5-methylpyridin-2-yloxy)-acetyl]-hydrazide (6f)

Yield $65 \%$, white solid $\mathrm{mp} ; 282-284^{\circ} \mathrm{C}$, IR $\left(\mathrm{cm}^{-1}\right)$ : 1635,1685(C=O), 3240-3460 (NH-NH); ${ }^{1} \mathrm{H}$
NMR $\left(\mathrm{CDCl}_{3}\right): \delta(\mathrm{ppm})=1.93\left(\mathrm{~s}, 3 \mathrm{H}, \mathrm{CH}_{3}\right), 3.51$ (s, 2H, $\left.\mathrm{CH}_{2}\right), 4.68\left(\mathrm{~s}, 2 \mathrm{H}, \mathrm{CH}_{2}\right), 6.28-7.65(\mathrm{~m}, 7 \mathrm{H}$, Ar), 10.32 (d, 2H, 2NH). LC-MS m/z = 334 Anal. Cal. for $\mathrm{C}_{16} \mathrm{H}_{16} \mathrm{CIN}_{3} \mathrm{O}_{3}: \mathrm{C}, 57.58 ; \mathrm{H}, 4.83$. Found: $\mathrm{C}, 57.67$; $\mathrm{H}, 4.35 \%$.

(3,4-Dichloro-phenyl)-acetic acid N'-[2-(5-methylpyridin-2-yloxy)-acetyl]-hydrazide (6g)

Yield $68 \%$, white solid $\mathrm{mp} ; 286-288^{\circ} \mathrm{C}$, IR $\left(\mathrm{cm}^{-1}\right)$ : 1645, $1690(\mathrm{C}=\mathrm{O}), 3230-3455(\mathrm{NH}-\mathrm{NH})$; ${ }^{1} \mathrm{H}$ NMR $\left(\mathrm{CDCl}_{3}\right): \delta(\mathrm{ppm})=1.98\left(\mathrm{~s}, 3 \mathrm{H}, \mathrm{CH}_{3}\right), 3.47$ (s, 2H, $\left.\mathrm{CH}_{2}\right), 4.50\left(\mathrm{~s}, 2 \mathrm{H}, \mathrm{CH}_{2}\right), 6.27-7.55(\mathrm{~m}, 6 \mathrm{H}$, Ar), $10.20(\mathrm{~d}, 2 \mathrm{H}, 2 \mathrm{NH}) \cdot{ }^{13} \mathrm{C} \mathrm{NMR}\left(100 \mathrm{MHz}, \mathrm{CDCl}_{3}\right)$ : $34.4,54.19,126.24,133.49,136.74,137.65$ 138.87, 143.36, 164.15. LC-MS $\mathrm{m} / \mathrm{z}=368$ Anal Cal. for $\mathrm{C}_{16} \mathrm{H}_{15} \mathrm{Cl}_{2} \mathrm{~N}_{3} \mathrm{O}_{3}: \mathrm{C}, 52.19 ; \mathrm{H}, 4$.11. Found: $\mathrm{C}$, 52.23; $\mathrm{H}, 4.06 \%$.

\section{4-Methoxy-benzoic acid N'-[2-(5-methyl-pyridin-} 2-yloxy)-acetyl]-hydrazide (6h)

Yield $64 \%$, white solid $\mathrm{mp} ; 274-276^{\circ} \mathrm{C}$, IR $\left(\mathrm{cm}^{-1}\right)$ : 1625, $1675(\mathrm{C}=\mathrm{O}), 3240-3460(\mathrm{NH}-\mathrm{NH})$; ${ }^{1} \mathrm{H} \mathrm{NMR}\left(\mathrm{CDCl}_{3}\right): \delta(\mathrm{ppm})=2.08\left(\mathrm{~s}, 3 \mathrm{H}, \mathrm{CH}_{3}\right), 3.24$ $\left(\mathrm{s}, 3 \mathrm{H}, \mathrm{CH}_{3}\right), 4.47\left(\mathrm{~s}, 2 \mathrm{H}, \mathrm{CH}_{2}\right), 6.33-7.70(\mathrm{~m}, 7 \mathrm{H}$, $\mathrm{Ar}), 10.28(\mathrm{~d}, 2 \mathrm{H}, 2 \mathrm{NH})$. LC-MS $\mathrm{m} / \mathrm{z}=316$ Anal. Cal. for $\mathrm{C}_{16} \mathrm{H}_{17} \mathrm{~N}_{3} \mathrm{O}_{4}: \mathrm{C}, 60.94 ; \mathrm{H}, 5.43$. Found: $\mathrm{C}, 61.01$; $\mathrm{H}, 5.32 \%$.

\section{RESULT AND DISCUSSION}

Synthesis of the target compounds 6a-h was performed according to the reactions illustrated in scheme 1. Initially, esterification of 8-hydroxy quinoline and 5-methyl-pyridin-2-ol 1a-b was performed by using chloroethyl acetate 2 in the presence of anhydrous potassium carbonate and dry acetone at reflux temperature to afford compounds 3a-b. These materials were stirred with hydrazine hydrate in ethanol to afford hydrazide derivatives $4 a-b$. Finally, the target derivatives $6 a-$ $\mathrm{h}$ were obtained in good yields by stirring of compounds $4 \mathrm{a}-\mathrm{b}$ with substituted carboxylic acids $5 \mathrm{a}-\mathrm{C}$ in the presence of $\mathrm{N}, \mathrm{N}, \mathrm{N}^{\prime}, \mathrm{N}^{\prime}$-tetramethyl-o(benzotriazol-1-yl) uranium tetrafluoroborate (TBTU) as a coupling agent and lutidine as a base in dry DCM at RT. The structure elucidations of the synthesized compounds were confirmed by IR, NMR, mass spectrometry and elemental analysis.

The spectra of the title compound 4 a were considered as a representative example of the 
series 4a-b. In IR spectra, the compound 4a showed band at 1720 and in between $3115-3225 \mathrm{~cm}^{-1}$ corresponding to carbonyl and $\mathrm{NH}-\mathrm{NH}_{2}$ stretching frequencies respectively. In ${ }^{1} \mathrm{H}$ NMR spectra of compound $4 \mathrm{a}$ showed one singlet at d 4.45 assigned to $\mathrm{NH}_{2}$ and broad singlet at $\mathrm{d} 4.71$ assigned to $\mathrm{OCH}_{2}$ protons, it also revealed broad singlet at d 9.41 assigned to amide proton as well as multiplet signals appeared in the range $d$ 7.218.87 for aromatic protons.

In IR spectra of compounds $6 a$ was confirmed by the appearance of one more carbonyl at $1685 \mathrm{~cm}^{-1}$ and disappearance of the $\mathrm{NH}_{2}$ absorption peak. In addition, ${ }^{1} \mathrm{H}$ NMR spectra showed disappearance of $\mathrm{NH}_{2}$ protons at 4.45 and an increase in one more $\mathrm{NH}$ proton and four aromatic protons with earlier aromatic proton peaks at d10.23 and 7.26-8.94 respectively, which clearly indicate the formation of compound 6a. The mass spectra of compound 6a gave significant stable $\left(\mathrm{M}^{+}\right)$ peak at $\mathrm{m} / \mathrm{z} 354$. Further, all the target compounds 6a-h were clearly confirmed by ${ }^{13} \mathrm{C}$ NMR.

\section{Molecular docking studies}

The molecular docking was performed and analyzed using Auto Dock 4.2. A Lamarkian genetic algorithm method implemented in the program suite was employed to identify appropriate binding modes and conformation of the ligand molecules. Gasteiger charges were added and the rotatable bonds were set by the Auto Dock tools and all torsions were allowed to rotate. Polar hydrogen atoms were added and Kollaman charges were assigned to the protein using Auto Dock tools (ADT). All the compounds $(6 a-h)$ were found to have minimum binding energy ranging from -6.29 to $-8.20 \mathrm{~kJ} / \mathrm{mol}$ with Aurora A kinase (PDB Code:3FDN).

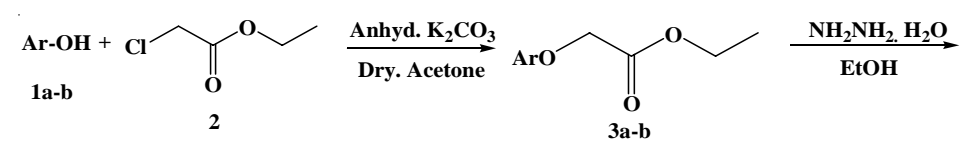

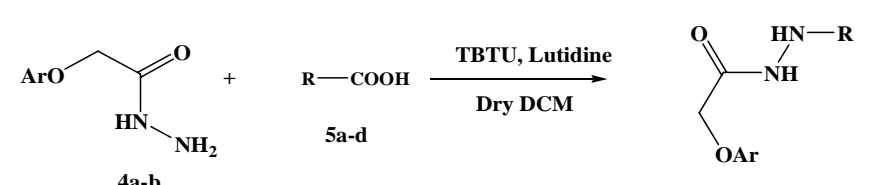

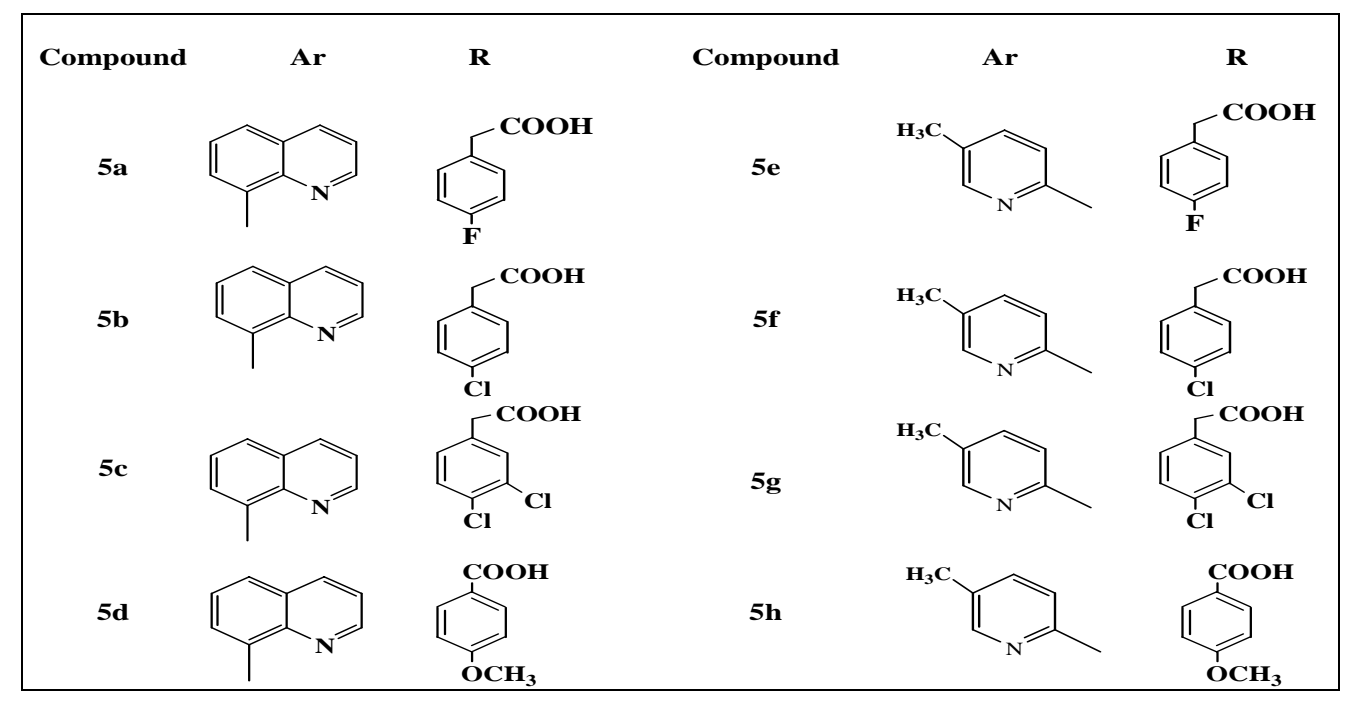

Scheme 1. Synthesis of quinoline and pyridine derivatives (6a-h) 
Table. 1: The dock score results of the different quinoline derivatives with Aurora A kinase target (PDB Code: 3FDN)

\begin{tabular}{lccccccc}
\hline $\begin{array}{l}\text { Com- } \\
\text { pounds }\end{array}$ & $\begin{array}{c}\text { Binding } \\
\text { Energy } \\
\left(\mathrm{kJ} \mathrm{mol}^{-1}\right)\end{array}$ & $\begin{array}{c}\text { Ligand } \\
\text { Efficiency }\end{array}$ & $\begin{array}{c}\text { Inhibition } \\
\text { Constant }\end{array}$ & $\begin{array}{c}\text { vdW+H- } \\
\text { bond+dissolve } \\
\text { energy }\end{array}$ & $\begin{array}{c}\text { No. of } \\
\mathrm{H}- \\
\text { bonds }\end{array}$ & $\begin{array}{c}\text { Bonding } \\
\text { residues }\end{array}$ & $\begin{array}{c}\text { Bond } \\
\text { Length } \\
(\AA)\end{array}$ \\
\hline $6 \mathrm{a}$ & -6.96 & -0.27 & 7.97 & -7.9 & 2 & $\begin{array}{c}\text { 3FDN:A:ARG137:HH22 } \\
\text { 3FDN:A: THR217:HN }\end{array}$ & $\begin{array}{c}1.958 \\
1.778\end{array}$ \\
$6 \mathrm{~b}$ & -7.73 & -0.3 & 2.15 & -9.04 & - & - & - \\
& & & & & & & \\
$6 \mathrm{c}$ & -8.2 & -0.3 & 978.84 & -9.12 & 1 & 3FDN:A: LYS162:HZ3 & 2.155 \\
$6 \mathrm{~d}$ & -7.64 & -0.29 & 2.51 & -8.99 & - & - & - \\
$6 \mathrm{e}$ & -6.29 & -0.27 & 24.63 & -6.93 & 1 & 3FDN:A: LYS162:HZ3 & 1.778 \\
$6 \mathrm{f}$ & -6.67 & -0.29 & 12.98 & -8.25 & 1 & 3FDN:A: LYS162:HZ3 & 2.171 \\
$6 \mathrm{~g}$ & -7.31 & -0.3 & 4.36 & -8.48 & 1 & 3FDN:A: ALA213:HN & 1.884 \\
$8 \mathrm{~h}$ & -6.63 & -0.29 & 13.9 & -7.99 & 2 & 3FDN:A:ARG137:HH22 & 2.052 \\
& & & & & & 3FDN:A: ALA213:HN & 1.859 \\
\hline
\end{tabular}

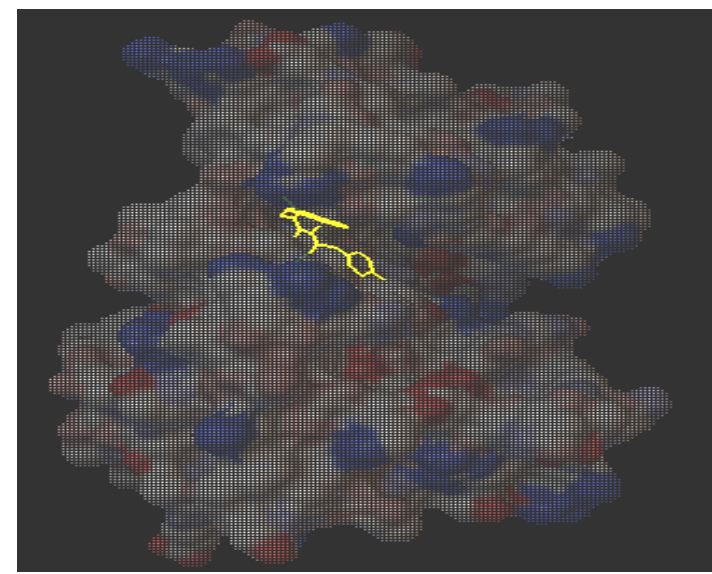

Enfolding of molecule $6 \mathrm{~b}$ in the active site pocket of Aurora Kinase

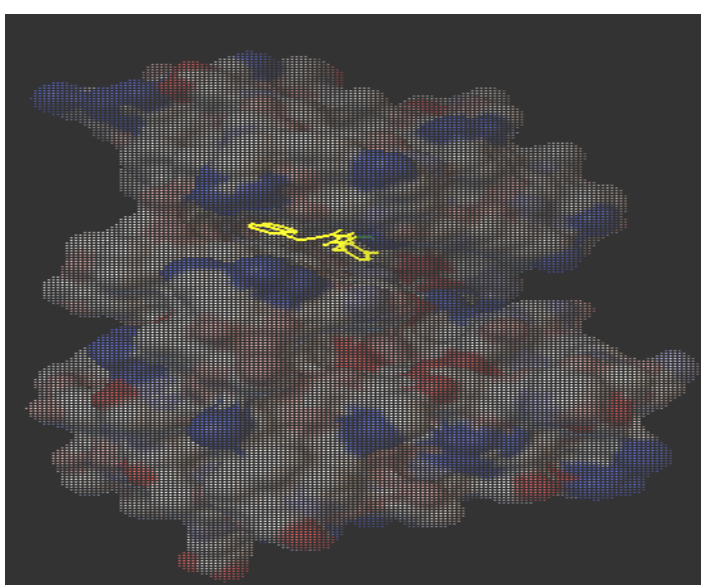

Enfolding of molecule $6 \mathrm{~d}$ in the active site pocket of Aurora Kinase

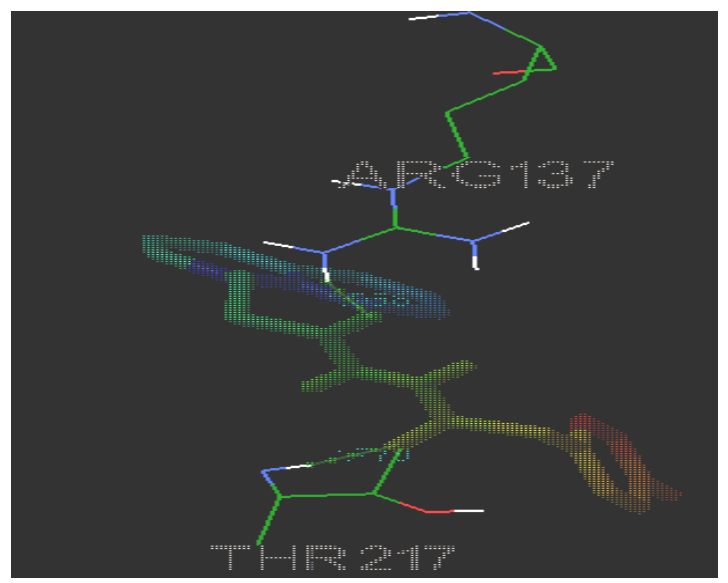

H-bond interaction of ligand molecule 6 a with 3FDN

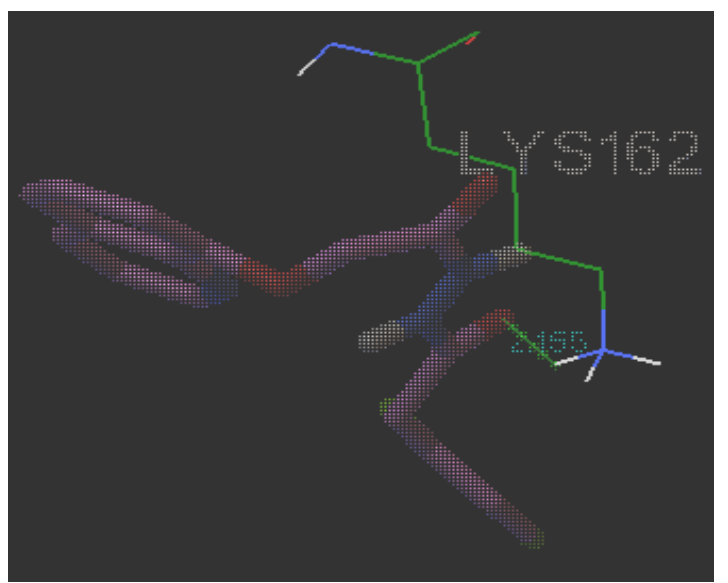

H-bond interaction of ligand molecule $6 \mathrm{c}$ with 3FDN 


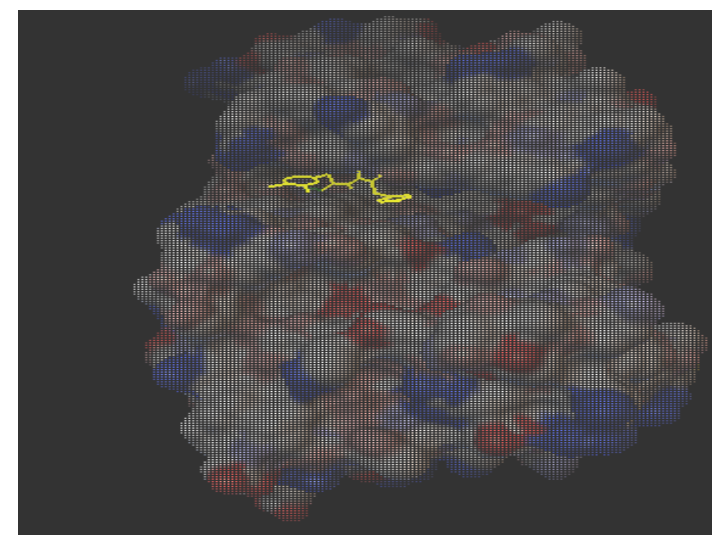

Enfolding of molecule $6 \mathrm{~g}$ in the active site pocket of Aurora Kinase

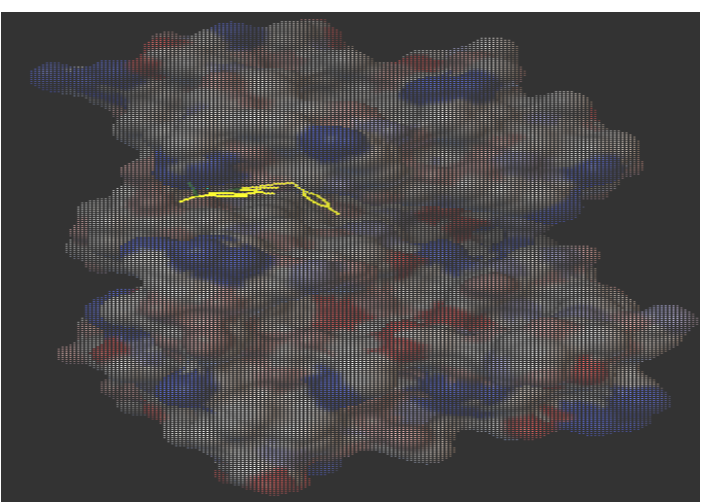

Enfolding of molecules $8 \mathrm{~h}$ in the active site pocket of Aurora Kinase

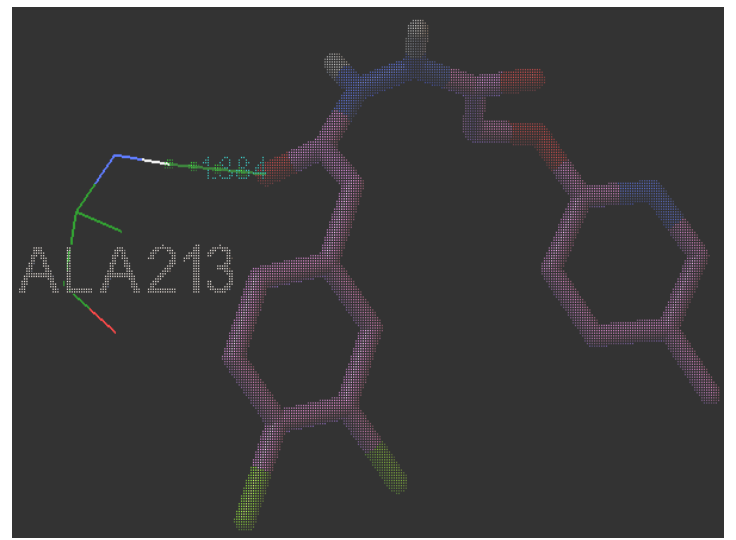

$\mathrm{H}$-bond interaction of ligand molecule $6 \mathrm{~g}$ with 3FDN

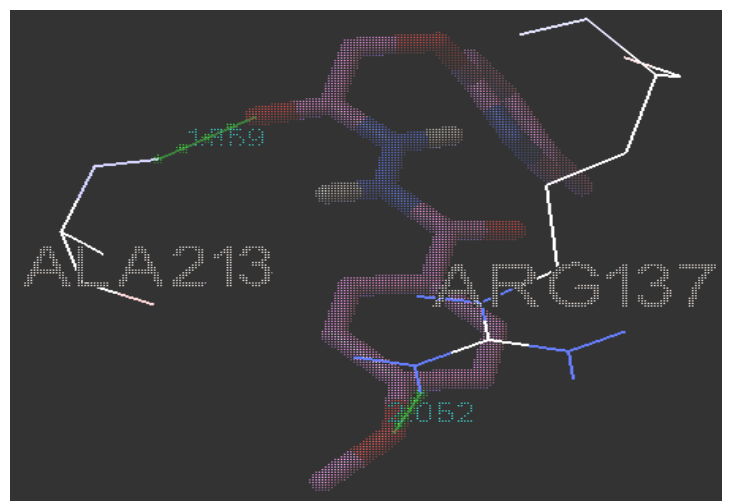

$\mathrm{H}$-bond interaction of ligand molecule $8 \mathrm{~h}$ with 3FDN

Fig.1 \& Fig.2

Among the molecules tested for docking study, (3,4-Dichloro-phenyl)-acetic acid N'-[2(quinolin-8-yloxy)-acetyl]-hydrazide (6c) showed minimum binding energy of $-8.20 \mathrm{~kJ} / \mathrm{mol}$ with ligand efficiency of -0.30 . Most of the residues, in nature. in close proximity to the inhibitor are hydrophobic in nature. The ligand molecules, $6 \mathrm{~b}, 6 \mathrm{~d}$ and $6 \mathrm{~g}$ revealed binding energy of $-7.73,-7.64$ and -7.31 $\mathrm{kJ} / \mathrm{mol}$, with ligand efficiency of $-0.30,-0.29$ and -0.30 , respectively. These molecules were completely wrapped by active site amino acid residues at the active site pocket region (Fig. 1). Similarly, molecules $6 \mathrm{a}, 6 \mathrm{c}$ and $6 \mathrm{~g}$ were found to show hydrogen bond interaction with active site amino acid residues Arg 137, Thr 217, Lys 162, Ala

213 and at a distance of (1.958 and 1.778), (2.155) and (1.884) $\AA$, respectively (Fig. 2). The docking study results showed that the molecules (6a-h) have good inhibition constant, $\mathrm{vdW}+\mathrm{H}$ bond + dissolve energy with best RMSD value.The details of docked score results of the molecules with Aurora A kinase (PDB Code:3FDN) are given in the Table 1 (see supplementary material).

\section{ACKNOWLEDGEMENTS}

The acknowledgement should be brief and must be written about the original supporters of the work and to the reputed institutions.

\section{REFERENCES}

1. Embrey, M.W.; Wai, J.S.; Funk, T.W.; Homnick, C.F.; Perlow, D.S.; Young, S.D.; Vacca, J.P.; Hazuda, D.J.; Felock, P.J.; Stillmock, K.A.;
Witmer, M.V.; Moyer, G.; Schleif, W.A.; Gabryelski, L.J.; Jin, L.; Chen, IWu.; Ellis, J.D.; Wong, B.K.; Lin, J.H.; Leonard, Y.M.; Tsou N.N.; 
Zhuang, L. Bioorg. Med. Chem. Lett. 2005, 15, 4550-4554.

2. Zhuang, L.; Wai, J.S.; Embrey, M.W.; Fisher, T.E.; Egbertson, M.S.; Payne, L.S Guare, J. P.; Vacca, J.P.; Hazuda, D.J.; Felock, P.J.; Wolfe, A.L.; Stillmock, K.A.; Witmer, M.V.; Moyer, G.; Schleif, W.A.; Gabryelski, L.J.; Leonard, Y.M.; Lynch, J.J.; Michelson, S.R.; Young, S.D. J. Med. Chem. 2003, 46, 453-456.

3. Atanasova, M.; Ilieva, S.; Galabov, B. Euro. J. Med. Chem. 2007, 42, 1184-1192.

4. Cope H, Mutter R, Heal W, Pascoe C, Brown P, Pratt S, Chen. B. Eur J Med Chem. 2006, 41, 1124-1143.

5. Mamladesai, S.N.; Katagi, M.S.; Khare, P.V.; Maddi, V.S.; Bhat, A.R. Indian J. Hetero. Chem. 2008, 17, 381-382.

6. Kamel, M.A.; Lobna, A.M.; El-Sayed, L.M.; Mohamed, H.I.; Rania B.H. Biorg Med. Chem. 2006, 14, 8675-8682.

7. Ola, E.I.; Sayed, A.; Fatma, E.M.; Shada, E.I.; Badr, A.A.; Maher, H.E. Indian J. Hetero. Chem. 2005, 14, 327-330.

8. Kansagra, B.P.; Bhatt, H.H.; Parikh, A.R. Indian J. Hetero. Chem. 2000, 10, 05-08.

9. $\quad$ Santhosh Kumar, S.; Ajay, N.; Tiwari, R.P.; Praveen. J. Indian J. Chem. 2006, 45, 17341739.

10. Amit, N.; Rahul, J. Indian J. Chem. 2008, 47, 117-128.

11. Vangapandu, S.; Jain, M.; Jain, R.; Kaur, S.; Singh, P.P. Bioorg Med Chem. 2004, 12, 2501- 2508.

12. Vikramdeep, M.; Amit, N.; Balasubramanian,
V.; Prakash, P.B.; Sarbjit, S.J.; Sukhraj K, Prati, P. S.; Rahul, J. Biorg Med. Chem. 2004, 12, 6465-6472.

13. Tseng, C.H.; Chen, Y.L.; Lu, P.J.; Yang, C.N.; Tzeng, C.C. Bioorg Med Chem. 2008, 16, 3153-3162.

14. Katarzyna, S.; Lukasz, K.S. Wojciech, S.J.; Joanna, W.; Marta, S.; Wanda, P.C. Exper. Therp. 2007, 61, 41-200.

15. Zhao, Y.L.; Chen, Y.L.; Chang, F.S.; Tzeng, C.C.; Eur J Med Chem. 2005, 40, 792-797.

16. Lazennec, G.; Richmond, A. Trends Mol Med. 2010, 16, 133-144.

17. Schwarz, M.K.; Wells, T.N. Nat Rev Drug Discov. 2002, 1, 347-358.

18. Al-Ghorbani, M.; Prabhu, T.; Gurupadaswamy, H.D.;Vigneshwaran, V.; Prabhakar. B.T.; Khanum, S. A. Bioorg. Chem, 2017, 71, 55-66.

19. Al-Ghorbani, M.; Prabhu, T.;, Vigneshwaran, V.; Gurupadaswamy, H.D.; Girish, V.; Prabhakar, B.T.; Khanum, S.A. Bioorg. Chem, 2016, 65, 73-81.

20. Al-Ghorbani, M.; Pavankumar, G.S.; Naveen, P.; Shamanth, H.G, Prabhakar, B.T.; Shaukath Ara Khanum, Bioorg. Chem, 2016, 65, 110-117.

21. Al-Ghorbani, M.; Vigneshwaran, V.; Lakshmi Ranganatha, V.; Prabhakar, B.T.; Khanum, S.A. Bioorg. Chem, 2015, 60, 136-146.

22. Al-Ghorbani M, Rekha N.D, Lakshmi Ranganatha V, Prashanth V, Veer aba sappagowda T, Khanum S.A, Russian J. Bioorg. Chem, 2015, 41, 4, 1-8. 